\title{
A low-drift, low-noise, multichannel dc voltage source for segmented-electrode Paul traps
}

Nikolai Beev, Julia-Aileen Fenske, Stephan Hannig, and Piet O. Schmidt

Citation: Review of Scientific Instruments 88, 054704 (2017); doi: 10.1063/1.4983925

View online: https://doi.org/10.1063/1.4983925

View Table of Contents: http://aip.scitation.org/toc/rsi/88/5

Published by the American Institute of Physics

\section{Articles you may be interested in}

Cryogenic setup for trapped ion quantum computing

Review of Scientific Instruments 87, 113103 (2016); 10.1063/1.4966970

A highly stable monolithic enhancement cavity for second harmonic generation in the ultraviolet Review of Scientific Instruments 89, 013106 (2018); 10.1063/1.5005515

Fast, precise, and widely tunable frequency control of an optical parametric oscillator referenced to a frequency comb

Review of Scientific Instruments 88, 033101 (2017); 10.1063/1.4977049

Noise characterization of analog to digital converters for amplitude and phase noise measurements Review of Scientific Instruments 88, 065108 (2017); 10.1063/1.4984948

High-precision, accurate optical frequency reference using a Fabry-Perót diode laser

Review of Scientific Instruments 88, 063101 (2017); 10.1063/1.4985544

Cryogenic mount for mirror and piezoelectric actuator for an optical cavity

Review of Scientific Instruments 88, 063104 (2017); 10.1063/1.4989404

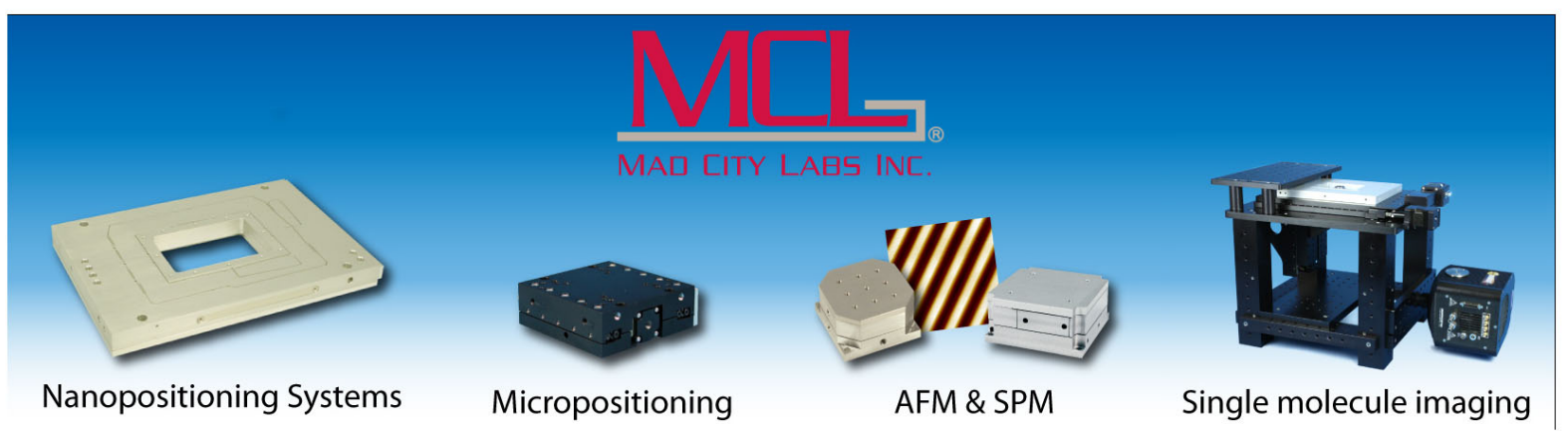




\title{
A low-drift, low-noise, multichannel dc voltage source for segmented-electrode Paul traps
}

\author{
Nikolai Beev, ${ }^{1, a), b)}$ Julia-Aileen Fenske, ${ }^{1}$ Stephan Hannig, ${ }^{1}$ and Piet O. Schmidt ${ }^{1,2}$ \\ ${ }^{1}$ Physikalisch-Technische Bundesanstalt, Bundesallee 100, 38116 Braunschweig, Germany \\ ${ }^{2}$ Institut für Quantenoptik, Leibniz Universität Hannover, Welfengarten 1, 30167 Hannover, Germany
}

(Received 28 February 2017; accepted 9 May 2017; published online 30 May 2017)

\begin{abstract}
We present the design, construction, and characterization of a multichannel, low-drift, low-noise dc voltage source specially designed for biasing the electrodes of segmented linear Paul traps. The system produces 20 output voltage pairs having a common-mode range of 0 to $+120 \mathrm{~V}$ with $3.7 \mathrm{mV} / \mathrm{LSB}$ (least significant bit) resolution and differential ranges of $\pm 5 \mathrm{~V}$ with $150 \mu \mathrm{V} / \mathrm{LSB}$ or $\pm 16 \mathrm{~V}$ with $610 \mu \mathrm{V} / \mathrm{LSB}$ resolution. All common-mode and differential voltages are independently controllable, and all pairs share the same ground reference. The measured drift of the voltages after warm-up is lower than 1 LSB peak-to-peak on the time scale of $2 \mathrm{~h}$. The noise of an output voltage measured with respect to ground is $<10 \mu \mathrm{V}_{\mathrm{RMS}}$ within $10 \mathrm{~Hz}-100 \mathrm{kHz}$, with spectral density lower than $3 \mathrm{nV} \mathrm{Hz}^{-1 / 2}$ above $50 \mathrm{kHz}$. The performance of the system is limited by the external commercial multichannel DAC unit NI 9264, and in principle, it is possible to achieve higher stability and lower noise with the same voltage ranges. The system has a compact, modular, and scalable architecture, having all parts except for the DAC chassis housed within a single 19" 3HE rack. Published by AIP Publishing. [http://dx.doi.org/10.1063/1.4983925]
\end{abstract}

\section{INTRODUCTION}

Trapped, laser-cooled ions present a powerful tool in experimental physics. ${ }^{1}$ Their application areas include optical clocks, ${ }^{2}$ search for variations in fundamental constants, ${ }^{3,4}$ quantum computing, ${ }^{5}$ and quantum simulation. ${ }^{6}$ An electric quadrupole trap, also known as Paul trap, uses a combination of rf and dc electric fields for ion confinement in three dimensions $\mathrm{s}^{7,8}$ with typical ion oscillation frequencies in the trap in the $\mathrm{MHz}$ range.

Laser cooling enables high-precision spectroscopy on trapped ions by reducing their random thermal motion. An important figure of merit for any trap is the heating rate $\dot{n}$, given in phonons per second $\left(\mathrm{s}^{-1}\right)$. It quantifies the strength of the coupling between the isolated trapped ion and the environment and depends on numerous factors of a fundamental and technical nature. ${ }^{9}$ A lower bound for the heating rate is given by fluctuating electric fields from thermal Johnson noise in the trap electrodes. Heating rates beyond the Johnson noise limit have been dubbed "anomalous" and their origin is still under investigation. ${ }^{9}$ Low heating rates are desirable, as they determine the coherence time of motional states, leading to smaller systematic shifts in clocks and longer coherence time in quantum gates. Linear Paul traps with segmented dc electrodes (Fig. 1) offer certain advantages, such as the ability to load ions in one region and then transport them for measurements in a "clean" part, where the anomalous heating rate caused by patch charges is expected to be significantly lower. Such traps also enable more sophisticated control of ion dynamics in advanced schemes, such as quantum logic

\footnotetext{
a) Author to whom correspondence should be addressed: nikolai.beev@ cern.ch. Telephone: +41 227675179.

b) Present Address: CERN, CH-1211 Geneva 23, Switzerland.
}

spectroscopy, where different ion species are co-trapped and their Coulomb interaction is exploited for sympathetic cooling, state preparation, and detection. ${ }^{1-13}$ Rapid shuttling of ions for quantum computing is yet another application area of segmented-electrode traps, ${ }^{5,14}$ and agile, low-voltage arbitrary waveform generators have been developed for this purpose. ${ }^{15}$ The traps are typically operated under ultra-high vacuum conditions to reduce collisions with background gas particles that would heat the ion(s), reorder ion strings, and can lead to the formation of molecules, such as hydrates.

Random fluctuations of the trapping potentials (i.e., externally induced electric field noise) are one possible source of ion heating. This effect can be suppressed by feeding stable, well-filtered voltages to the trap's dc electrodes. If the null of the pseudopotential generated by the rf field and the null of the effective dc field do not overlap, this leads to a parasitic effect known as micromotion. ${ }^{16,17}$ While micromotion usually does not cause heating directly, ${ }^{9}$ it is a limiting factor in optical clock experiments due to the introduced second-order Doppler and rf-induced ac Stark shifts. ${ }^{2}$ Typically, traps have provisions for compensating it by fine adjustment of the ion position through the application of dc voltages on auxiliary electrodes (Fig. 1).

Various types of stable, low-noise voltage sources exist, each having characteristic advantages and disadvantages. Electrochemical batteries have inherently low noise down to very low frequencies ${ }^{18}$ and offer galvanic isolation, but to achieve sufficiently high voltages for average trap geometries, it is necessary to connect many cells in series. Furthermore, due to their limited lifespan and self-discharge characteristics, batteries have to be monitored and regularly replaced. An illustrative example is the ultra-stable voltage source for biasing the ring electrode of a Penning trap described in Ref. 19, which is based on a 66-element battery of 


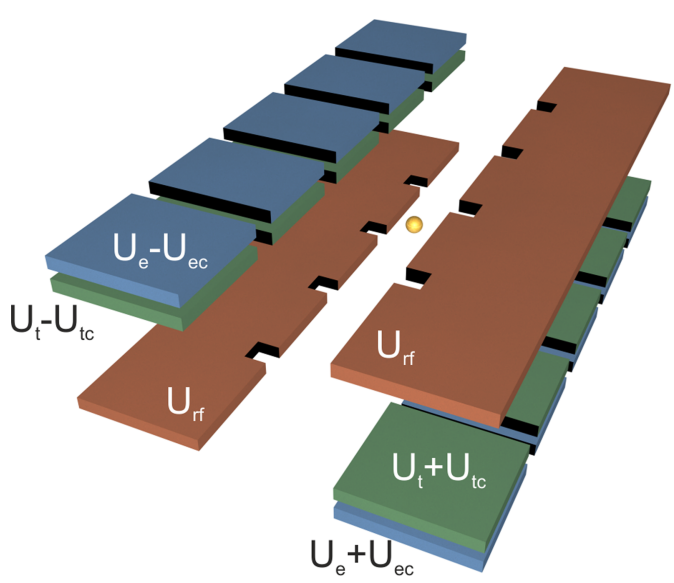

FIG. 1. Segmented linear Paul trap. ${ }^{10}$ The red electrodes are connected to a rf voltage $U_{\text {rf }}$ with respect to all other electrodes. They produce a $2 \mathrm{D}$ radial quadrupole field for the radial confinement of the ion. The green and the blue segmented electrodes are connected to dc voltages that can be set for every electrode pair individually. The voltages $U_{t}$ provide axial confinement and positioning of the ion, here symbolized by the yellow sphere. Applying differential voltages $U_{e c}$ and $U_{t c}$ to opposing electrodes allows controlling the position of the ion in the radial direction and thus the minimization of micromotion.

unsaturated Weston cells. The authors discuss the disadvantages of the approach and then proceed to describe a more standard and modern solution employing series-connected buried-Zener voltage references that achieve a comparable level of stability in the tens of ppb on the time scale of hours. ${ }^{19}$ The requirements for voltage supplies in Penning trap experiments are unusually high due to specifics not shared by Paul traps.

Switching dc-dc converters are extremely common and flexible. Step-up variants with galvanic isolation and multiple output voltages are readily available. However, they tend to be noisy (Ref. 20, pp. 580, 635-636), often having multiple spurious peaks in the spectrum extending to high frequencies, where the parasitic feedthrough coupling degrades the performance of passive filters. Moreover, switching converters are often a source of radiated electromagnetic interference (EMI), which should be avoided in sensitive laboratory environments. Linear voltage supplies can be designed for low noise and low EMI impact, at the cost of high power dissipation and large physical size. For specific applications with very high requirements, special linear dc-dc converters operating on batteries have been developed. ${ }^{21}$ Low-noise, high-voltage converters also count on linear circuit solutions, such as the resonant Royer topology. ${ }^{22}$ A flexible, multi-channel supply designed for a low-noise and high-stability application typically contains multiple functional blocks, such as converters, regulators, reference voltage sources, amplifiers, and filters. ${ }^{23}$

The design of electronic devices with highly stable dc characteristics calls for the careful selection of elements, circuit topologies, and methods of alleviating numerous influences. Virtually, all passive and active components exhibit parametric temperature dependencies, aging, and noise of various origins. The circuit performance could also degrade due to external factors, such as air flow, mechanical stress, vibration, humidity, surface contamination, and the coupling of EMI [Refs. 24 and 25 (pp. 301-303)].

\section{SYSTEM DESIGN}

\section{A. Design goals}

The primary design criteria for the considered application are resolution, stability, and low noise of the output voltages. Absolute accuracy is not required, as the exact levels are not known a priori but are instead adjusted according to experimental results. High resolution is required, in order to compensate small imbalances in the electrode geometry due to fabrication tolerances, and stray charges, while stability is needed to avoid variations in the experimental parameters during measurement sessions. The dynamic performance is not an issue for the foreseen clock application, as the voltages only need to vary quasi-statically during ion transport or tuning operations but not during actual spectroscopy sequences. The quantitative resolution and stability goals were estimated conservatively at the $20 \mathrm{ppm}$ level ( $\approx 16$ bits), and the performance characteristics of common multichannel commercial 16-bit DAC units were used as a guideline.

The trapping dc electrode pairs are voltage-biased in such a way that the common-mode voltage $U_{t, k}$, where $k$ denotes the electrode position, determines the ion's axial confinement (oscillation frequency in the harmonic trap), while the differential-mode voltage $U_{t c, k}$ together with the voltage $U_{e c}$ determines its position (Fig. 1) to compensate micromotion. This scheme is well suited for the implementation of separate, independent controls. Since the outer micromotion compensation electrodes are shielded by the main trapping electrodes, an extended range is needed for the compensation voltages $U_{e c}$.

Electric field spectral components near the ion's trap frequency couple strongly to the motional degrees of freedom of the ions, causing heating. ${ }^{9}$ This mechanism imposes strict noise requirements on the system. In a segmented trap, these requirements are exacerbated by the fact that the ions are in proximity to a number of biased electrodes, causing them to experience the combined effect of numerous independent noise sources. We have thus treated the voltages for trapping and for micromotion compensation with equal attention, regarding both stability and noise.

Provided that external EMI pickup is adequately suppressed by shielding and decoupling, the dominant source of short-term voltage fluctuations in any active voltage source is electronic noise generated within the circuits. The objective is to start with a low-noise design and apply further low-pass filtering at the outputs. Since the trap frequencies are typically in the hundreds of $\mathrm{kHz}$ to $\mathrm{MHz}$ range, first- or second-order low-pass RC filters with cutoff frequency $f_{c}<100 \mathrm{~Hz}$ allow sufficient bandwidth for roll-off and high attenuation at the bands of interest.

While carefully avoiding ion heating by unwanted noise, a required system feature is the provision for controlled excitation of the trapped ions. This is commonly achieved by feeding a harmonic rf signal from a generator through a bias tee-a three-port circuit that combines ac and dc signals at a single output, while keeping the inputs mutually isolated. For the given application, it does not need to have very flat frequency response or high bandwidth, but it must tolerate the high dc voltages. In our system, a $+20 \mathrm{dBm}$ ac signal applied to the bias tee is required to provide sufficient rf voltage through the 
TABLE I. The characteristics of the NI 9264 DAC module. ${ }^{26}$

\begin{tabular}{lc}
\hline \hline Number of channels & 16 \\
Voltage range & $\pm 10,5 \mathrm{~V}$ \\
Sample rate & $25 \mathrm{kS} / \mathrm{s} / \mathrm{ch}$ \\
Resolution & $16 \mathrm{bits}$ \\
Gain accuracy & $0,05 \%$ \\
Offset accuracy $^{\mathrm{a}}$ & $5,3 \mathrm{mV}$ \\
Offset drift $^{\text {Gain drift }}$ & $80 \mu \mathrm{V} /{ }^{\circ} \mathrm{C}$ \\
Noise $^{\mathrm{b}}$ & $6 \mathrm{ppm} /{ }^{\circ} \mathrm{C}$ \\
& $500 \mu \mathrm{V}_{\text {RMS }}$ \\
\hline \hline
\end{tabular}

${ }^{\mathrm{a}}$ Calibrated, $\mathrm{T}=25^{\circ} \mathrm{C}$.

${ }^{\mathrm{b}}$ Given within an unspecified bandwidth.

low-pass RC filters to an electrode of the trap for a controlled excitation of the secular motion of the ion. The galvanic isolation of the ac signal is desirable in order to prevent the coupling of EMI through cable pickup or ground loops.

Long-term voltage variations known as drifts can originate from leakage currents on contaminated surfaces, mechanical stress, parasitic electrochemical cells, and, most importantly, temperature variations - either due to the direct temperature dependence of parameters (resistance, offset voltage, etc.) or through secondary effects, such as thermal electromotive forces (EMFs) across parasitic thermocouple junctions, e.g., solder joints (Ref. 25, pp. 265-268). While the system is to be operated in a laboratory environment with well-stabilized ambient temperature (peak-to-peak fluctuations below $0.2^{\circ} \mathrm{C}$ ), adequate board- and module-level thermal management must ensure that the heat generated within the elements is properly dissipated. We have opted for fully enclosing the circuit boards in order to provide good EMI shielding and protection against air flow, contamination, and humidity. We have considered drifts on the time scale of hours, which is the typical duration of experimental sessions. Issues related to component aging and stability on longer time scales are beyond the scope of this work.

\section{B. Architecture overview}

The system was designed to interface to a computercontrolled multichannel DAC unit. A National Instruments universal serial bus (USB)-connected chassis (type CDAQ9174) housing three NI 9264 modules ${ }^{26}$ was selected. The most relevant characteristics of these modules are given in Table I.

The block diagram of a single voltage pair channel is shown in Fig. 2. The entire system consists of twenty such channels, each having two input control voltages and two high-voltage (HV) outputs. The controls set independently the common-mode and differential output voltages. For all channels, the common-mode gain is 12 , yielding an output voltage range of $0-+120 \mathrm{~V}$. The differential output voltages are stabilized using feedback loops. All input and output voltages are referenced to a single ground potential, and all channels are powered from the same power supply rails. A low-voltage monitor port is available, where the output voltages are scaled down by 100:1 resistive dividers. In order to avoid amplifying broadband noise from the NI 9164 modules, the control voltages $V_{C M}$ and $V_{D I F F}$ are pre-filtered using single-pole lowpass filters with $f_{c}<10 \mathrm{~Hz}$. Low-pass filters are also present at the circuit outputs and physically close to the trap electrodes. The on-chip capacitors placed near the electrodes also serve as shunts for the inadvertently coupled rf trap drive.

\section{Composite amplifiers}

The use of HV monolithic operational amplifiers greatly reduces design complexity, component count, and circuitboard footprint in comparison to discrete-element solutions. Unfortunately, the dc performance of such amplifiers in a standalone configuration is not adequate for the given task. We have therefore utilized the Apex Microtechnology PA340 27 HV operational amplifier as a voltage-boosting output stage within a composite amplifier (Ref. 20, pp. 332-333) with a precision low-voltage input stage [Fig. 3(a)].

A non-critical feedback loop closed around the HV amplifier sets its noninverting gain to 12 , covering the output range up to $120 \mathrm{~V}$ for a $10 \mathrm{~V}$ input signal. The HV output is then sampled by a separate precision resistive network and fed back to the first stage, which consists of a conventional low-voltage precision operational amplifier type AD8675. ${ }^{28}$ The overall gain set by the precision network equals that of the HV booster, forcing the first stage to operate at near-unity gain. External

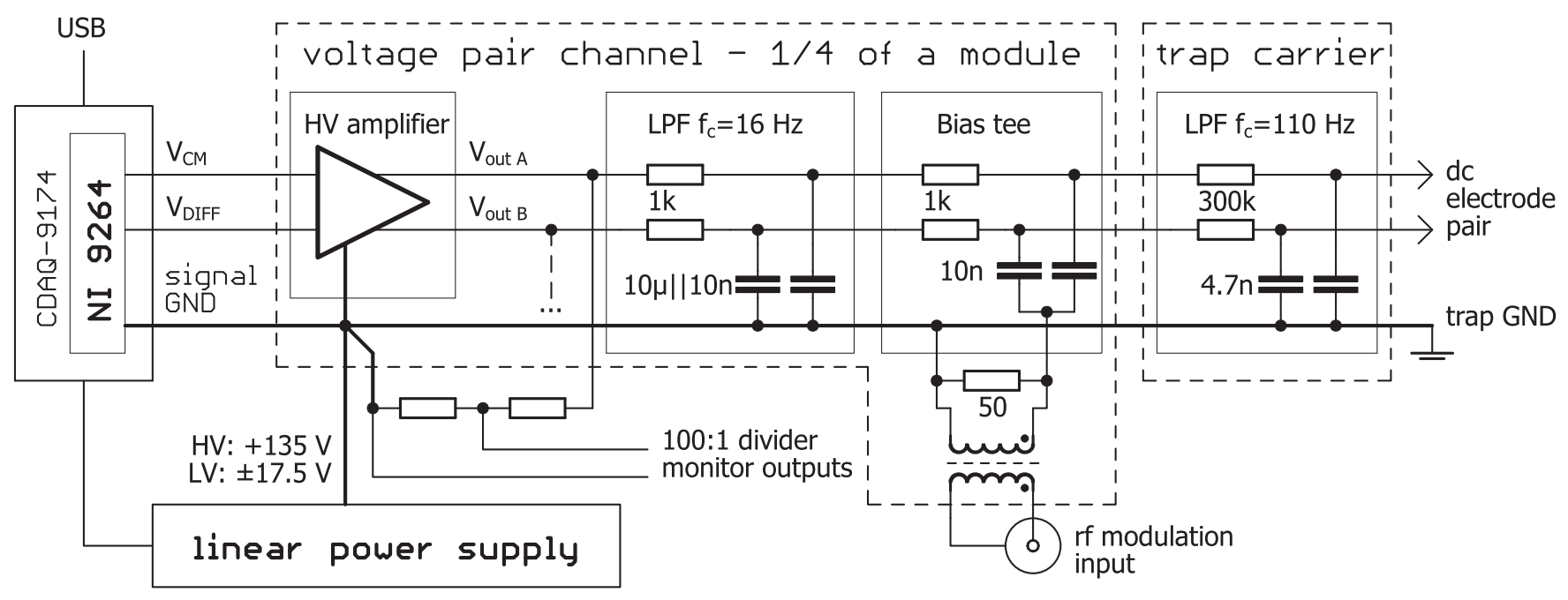

FIG. 2. System-level block diagram showing one voltage pair channel. 


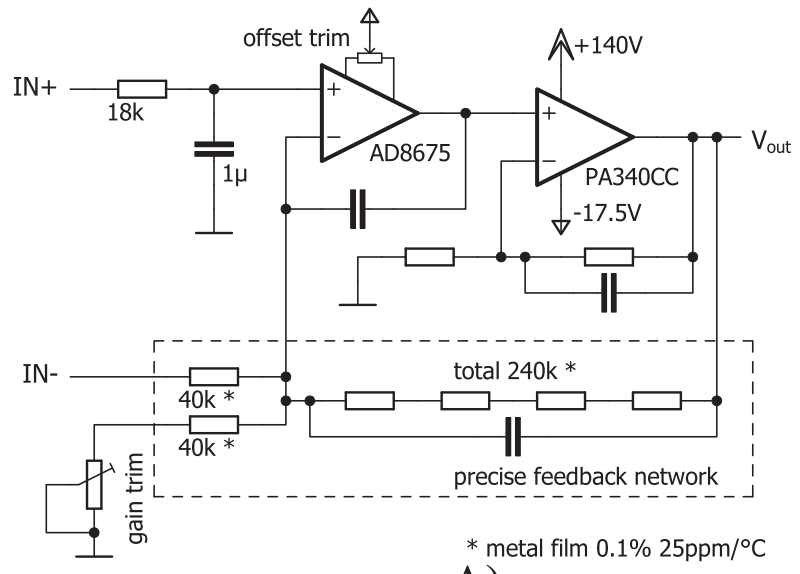

A)

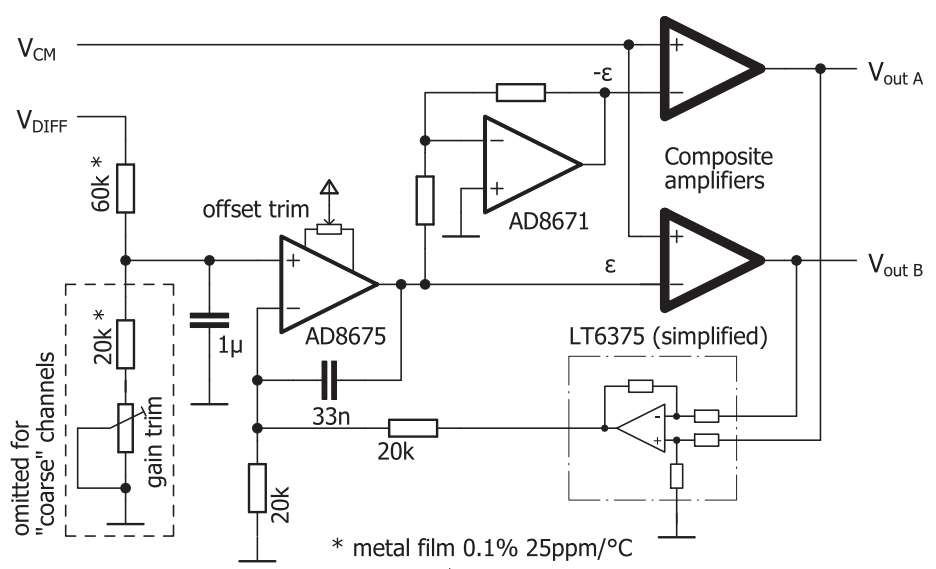

B)

FIG. 3. A simplified schematic diagram of (a) high-voltage composite amplifier and (b) voltage pair channel with differential feedback loop. $\epsilon$ denotes the error signal, which is proportional to $V_{D I F F}$.

compensation is necessary for the stability of this configuration (Ref. 25, pp. 341-342). Since only near-dc operation is required, we have overcompensated the amplifier in order to reduce its noise bandwidth ${ }^{25}$ (ch. 10).

The precision feedback network consists of metal-film resistors having $0.1 \%$ initial tolerance and $25 \mathrm{ppm} /{ }^{\circ} \mathrm{C}$ temperature coefficient of resistance. Resistors of this type have low excess $1 / f$ noise, ${ }^{29}$ which is bias-dependent and should be considered where high voltages are encountered. Multiple resistors are connected in series to alleviate the effects of Joule heating and to further improve the initial tolerances statistically. For a fixed gain, determined by the ratio of feedback resistors, the overall resistance level is a trade-off between noise and power dissipation. In the limit of very low resistances, the voltage noise $e_{n}$ of the first-stage amplifier dominates and noise at the output of the amplifier is minimized. However, the current through the feedback network and the power dissipation in it become intolerably high for the full range of output voltages. The opposite case of very high resistances is also undesirable, as the output noise increases, this time dominated by the back-action of the amplifier's current noise $i_{n}$. At the resistance noise optimum of the amplifier [Eq. (1)], the contributions of the voltage and current noise generators are equal, while the total output noise is dominated by Johnson noise of the resistors ${ }^{20}$ (p. 526)

$$
R_{\text {opt }}=\frac{e_{n}}{i_{n}}=\frac{2,8 \cdot 10^{-9}}{0,3 \cdot 10^{-12}}=9300 \Omega \text {. }
$$

The chosen values [Fig. 3(a)] result in a dissipation $\mathrm{P} \approx 55 \mathrm{~mW}$ in the resistive feedback network at maximum output voltage, while the output noise is still resistor-dominated.

Note that to maintain the gain and offset accuracy, both the inverting and non-inverting inputs of the described composite amplifier must be driven from low-impedance sources. The $18 \mathrm{k} \Omega$ resistor in the $\mathrm{RC}$ filter at the noninverting input is dimensioned to balance the offset due the input bias currents of the AD8675. More important is the case of the inverting input, which forms the part of the precision gain-setting network. If left open or driven by a very high impedance, the non-inverting gain of the amplifier would be twice lower. In order to have the desired gain of 12, this input should be either grounded or driven by a low impedance, e.g., the output of an operational amplifier.

\section{Differential voltage feedback loop}

To set and maintain the output differential voltage with high precision within a channel, a feedback loop was implemented. The circuit topology is shown in Fig. 3(b). Integrated difference amplifiers exist in variants that tolerate input common-mode voltages far outside their supply rails. In addition, they contain matched resistors with excellent tracking characteristics and typically a fixed differential-tosingle-ended gain with well-specified accuracy. We have chosen the LT6375A amplifier ${ }^{30}$ by Linear Technology for its common-mode range of $\pm 270 \mathrm{~V}$, high common-mode rejection (minimum $97 \mathrm{~dB}$ ), high gain stability, and low offset drift.

The difference amplifier samples the two high-voltage outputs $V_{\text {out } A}$ and $V_{\text {out B }}$ directly and produces a groundreferenced feedback signal $V=\left(V_{\text {out } A}-V_{\text {out } B}\right) / 2$. The differential set point is subtracted from this voltage by the error amplifier to produce the error signal $\varepsilon$, which goes to the inverting input of one of the composite amplifiers. An inverted replica $-\varepsilon$ is fed to the other composite amplifier in a symmetric manner. At low frequencies approaching dc, the open-loop gain increases at a rate of $20 \mathrm{~dB} /$ decade due to the single-pole integrator action of the error amplifier. In the closed loop, the residual static error due to the finite dc open-loop gain of the error amplifier $A_{0} \approx 120 \mathrm{~dB}$ is sufficiently small and can be ignored. We can thus describe the loop as a servo mechanism that locks together the two output voltages $V_{\text {out } A}$ and $V_{\text {out B }}$ nearly perfectly at dc and imposes a diminishing cross correlation until high frequencies, where the two voltages become independent again.

There are two types of channels based on the differentialmode voltage range- -fine" and "coarse." "Fine" channels have a range of $\pm 5 \mathrm{~V}$ produced by scaling down the $V_{D I F F}$ control signal from the NI 9264 output by a precision 4:1 divider and using the result as the set point voltage. The "coarse" channels do not have the divider and thus have a wider range of $\pm 16 \mathrm{~V}$, limited by the low-voltage supply rails. 
TABLE II. Estimated output dc static errors and temperature-dependent drift terms.

\begin{tabular}{lcccccc}
\hline \hline $\begin{array}{l}\text { Functional } \\
\text { block }\end{array}$ & Device & $\begin{array}{c}\text { Offset voltage } \\
\mathrm{V}_{\mathrm{OS}}(\mu \mathrm{V})\end{array}$ & $\begin{array}{c}\text { Gain error } \\
\Delta \mathrm{G}(\mathrm{ppm})\end{array}$ & $\begin{array}{c}\text { Offset voltage } \\
\text { drift } \Delta \mathrm{V}_{\mathrm{OS}} / \Delta \mathrm{T} \\
\left(\mu \mathrm{V} /{ }^{\circ} \mathrm{C}\right)\end{array}$ & $\begin{array}{c}\text { Gain drift } \\
\Delta \mathrm{G} / \Delta \mathrm{T} \\
\left(\mathrm{ppm} /{ }^{\circ} \mathrm{C}\right)\end{array}$ & $\begin{array}{c}\mathrm{dc} \mathrm{CMR} \\
(\mathrm{ppm})\end{array}$ \\
\hline Composite amplifier & AD8675 & 120 & $2000^{\mathrm{a}}$ & 2,4 & $50^{\mathrm{a}}$ & $<1$ \\
$\begin{array}{l}\text { Difference amplifier } \\
\text { Error amplifier }\end{array}$ & LT6375A & 50 & 4 & 2 & 0,1 & 5 \\
\hline \hline
\end{tabular}

${ }^{\mathrm{a}}$ Estimated worst-case for $\Delta \mathrm{R} / \mathrm{R}=0.1 \%, \mathrm{dR} / \mathrm{dT}=25 \mathrm{ppm} /{ }^{\circ} \mathrm{C}$.

\section{E. Error estimates}

The complexity of the design precludes full system-level error analysis, so we have instead chosen to focus on critical nodes and components. In Table II we give estimates of the most important output-referred errors for the principal amplifier stages, calculated from typical input-referred values given by the manufacturers. ${ }^{28,30}$ These estimates are separate - they do not reflect interactions, such as the operation of the differential feedback loop.

Apart from the basic distinction between initial static errors and drift terms, one also notes the uncertainties of the two independent parameters that define a linear transfer function - offset and gain. Nonlinearity terms are ignored, with the exception of common-mode rejection (CMR) errors. In the case of the difference amplifier, they represent a cross term between the $V_{C M}$ and $V_{D I F F}$ setting voltages and cannot be compensated by gain or offset trimming. Note that the numbers given in Table II represent typical values or worst-case estimates, while in reality, most parameters exhibit statistical distributions that occasionally yield parts with nearly ideal characteristics.

\section{F. Filters and bias tees}

Each output voltage is filtered to ground by a single-pole $\mathrm{RC}$ filter with $f_{c} \approx 16 \mathrm{~Hz}$. Two capacitors are connected in parallel in order to provide both high capacitance $(10 \mu \mathrm{F})$ and low shunt impedance at frequencies above the self-resonance of the large-value capacitor. We have used $10 \mu \mathrm{F} 250 \mathrm{~V}$ polyester and $10 \mathrm{nF} 250 \mathrm{~V}$ NP0 ceramic capacitors.

The bias tees use $1 \mathrm{k} \Omega$ resistors to isolate the dc input and $10 \mathrm{nF} 250 \mathrm{~V}$ NP0 capacitors to couple the ac signal. It is not necessary to use inductors, as the dc path is essentially unloaded and only needs to source current for charging the capacitors near the trap and the stray wiring capacitances. A 1:1 broadband signal transformer (MiniCircuits type T1-1T) is used for the galvanic isolation of the external rf input. The primary side of the transformer is grounded to the rack chassis through the coaxial connector, while the secondary side is referenced to the "floating" signal ground. The ac signal is fed in-phase to the two voltage lines in the pair, i.e., it excites the common-mode voltage.

\section{G. Layout, shielding, grounding, power supply, and construction}

The 20 voltage pair channels are divided into 5 units, each one consisting of two circuit boards housed together in an aluminum shielding box. The main board (Fig. 4) contains all active circuits, while the second board contains the output passive filters and bias tees. Both circuit boards have standard Eurocard size of $160 \times 100 \mathrm{~mm}$. A short ribbon cable provides the connection between the two circuit boards within the shielding box. All input and supply voltages are fed to the main board through the backplane connector. Additionally, the unfiltered output voltages are available on the same connector for monitoring purposes. The voltages from the outputs of the filters and bias tees are taken to a 9-pin D-SUB connector on the front panel of the module. Each unit contains two "fine" and two "coarse" channels, and all units are functionally identical and interchangeable.

The main board is realized as a 4-layer design. One of the inner layers is a solid ground plane that doubles as an isothermal heat sink. Multiple large copper areas in other layers serve the same purpose. The goal of this thermal design is to minimize temperature gradients across the board, particularly at the locations of the difference amplifiers. The HV operational amplifiers have additional heat sinks visible on the top side of the board (Fig. 4). Following the reflow soldering of all surfacemounted components, the boards were washed with ethanol in order to remove the soldering flux residue. This measure was necessary to avoid leakage currents from high-voltage points to sensitive high-impedance nodes like amplifier stage inputs.

The supply voltages for all parts in the system, including the NI 9264 modules, are provided by linear, regulated power supplies. Their secondary sides are "floating," i.e., not grounded to the rack chassis. The power supply and ground leads of each module are separately wired and connected together at a single point in a "star" configuration in order to

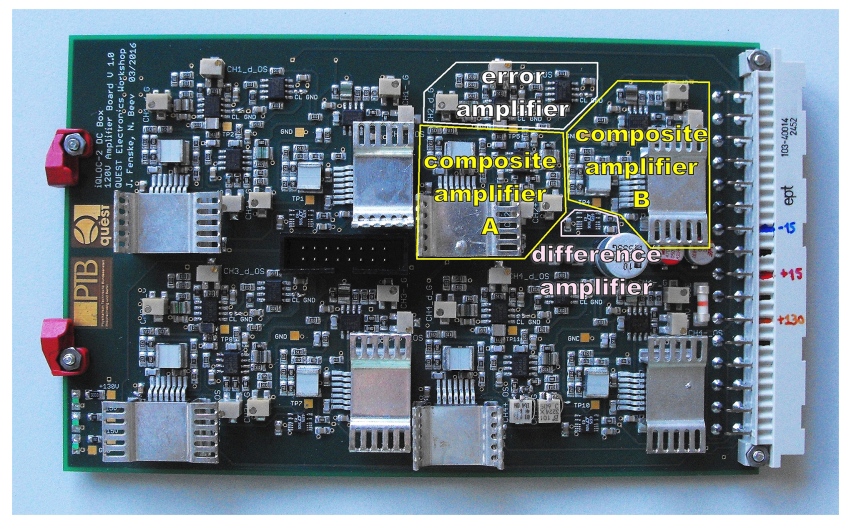

FIG. 4. Photograph of the Eurocard-sized $(160 \times 100 \mathrm{~mm})$ circuit board with four high-voltage pair channels. The principal blocks of one channel are shown: composite amplifiers, difference amplifier, and error amplifier. 

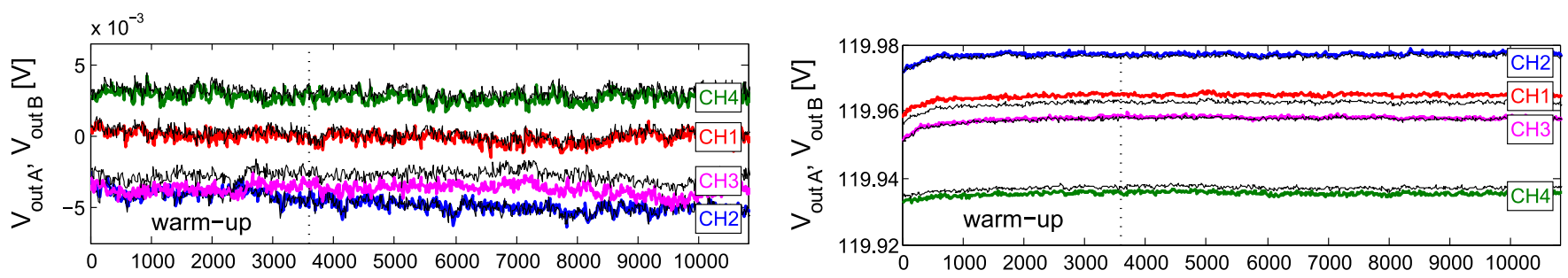

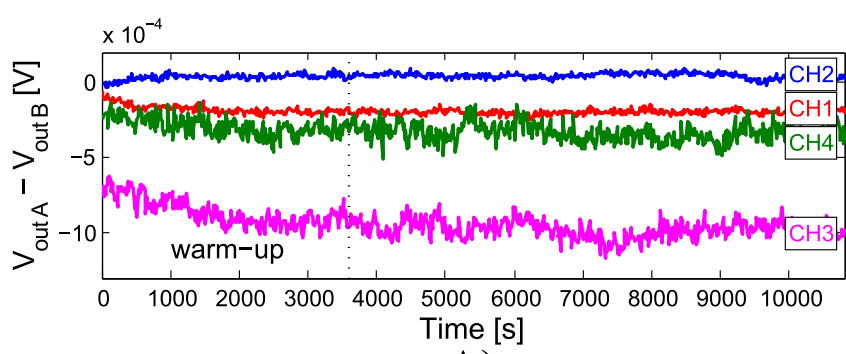

A)

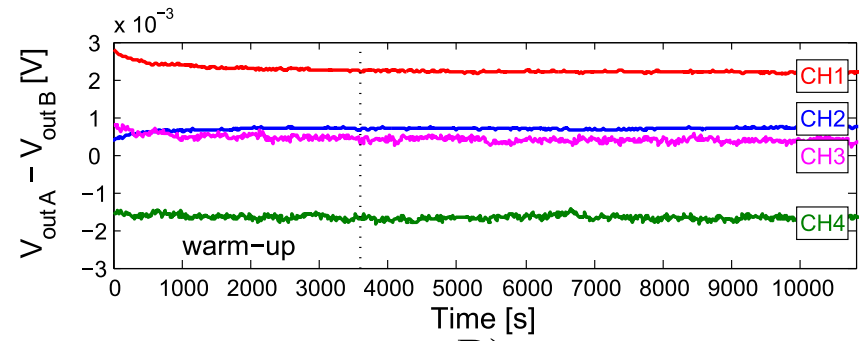

B)

FIG. 5. Time series plot showing the 8 ground-referenced and 4 differential voltages measured from a single unit (module no. 2) for (a) $V_{\text {out }} A=V_{\text {out }} B$ $=0 \mathrm{~V}$ and (b) $V_{\text {out } A}=V_{\text {out } B}=120 \mathrm{~V}$.

avoid shared impedance paths and voltage drops across ground leads. The rack chassis is hard-grounded to the mains inlet safety earth. Everything, apart from the CDAQ 9174 chassis, is housed within the same 19" $3 \mathrm{HE}$ rack. Connections to the NI 9264 units are made using shielded cables with 37-pin DSUB connectors. The outputs are also connected to the vacuum chamber feed-throughs using shielded cables and connectors.

\section{PERFORMANCE RESULTS}

All 20 voltage pair channels were characterized under identical conditions using an automated test bench, controlled by a National Instruments LabView program. The input control voltages were generated by the NI 9264 DAC modules. For the measurement shown in Fig. 7, the control voltages were either $0 \mathrm{~V}$ (shorted inputs) or a stable $10 \mathrm{~V}$ generated by a circuit utilizing a low-noise, low-drift LT1027 (Ref. 20, p. 545).

The outputs were switched through relays and measured using an Agilent 34410A 6,5-digit benchtop multimeter. The instrument was operated in auto-ranging mode and was read out using the general purpose interface bus (GPIB) interface.
The two output voltages in each channel were first measured against the ground reference then differentially. This scheme enables the high-resolution measurement of the differential voltages for any common-mode setting.

Measurements on all 4 channels within a single module unit were performed in time-interleaved manner. Each measurement had a dwell time of $1 \mathrm{~s}$ during which 10 samples were recorded. The first two of them were discarded to avoid settling errors, and the remaining 8 were averaged, yielding an effective sampling aperture time $\tau=800 \mathrm{~ms}$ and sampling rate $F_{S}=0,0833 \mathrm{~S} / \mathrm{s}$ for each measured voltage.

The system was switched off and allowed to reach equilibrium with the ambient room temperature between consecutive measurement sessions in order to consistently record its warmup behavior. Forced-air cooling was provided continuously by three $12 \mathrm{~V}$ computer fans having an air flow rate of $69 \mathrm{~m}^{3} / \mathrm{h}$, placed directly on top of the rack. The total power dissipation of the system was monitored at the mains inlet-it varied between $40 \mathrm{~W}$ (all output voltages at $0 \mathrm{~V}$ ) and $60 \mathrm{~W}$ (all output voltages at $120 \mathrm{~V}$ ). The latter situation is unlikely in normal operation but provides a valuable limit test. We found that forced-air cooling of the fully enclosed modules reduced the

TABLE III. The measured performance results for all 20 channels.

\begin{tabular}{lcccc}
\hline \hline Measurement & $\mathrm{V}_{\text {out }}{ }^{\mathrm{a}}(\mathrm{V})$ & Minimum & Median & Maximum \\
\hline Ground-referenced voltage warm-up drift $(\mathrm{mV})$ & 0 & $-3,237$ & $-0,798$ & 2,333 \\
& 120 & 2,255 & 5,691 & 9,114 \\
Ground-referenced voltage 2 h drift $\left(\mathrm{mV}_{\mathrm{p}-\mathrm{p}}\right)$ & 0 and 120 & 1,806 & 2,477 & 3,412 \\
Differential voltage warm-up drift ("fine" channels) $(\mu \mathrm{V})$ & 0 & -126 & 14 & 111 \\
& 120 & -570 & -24 & 281 \\
Differential voltage warm-up drift ("coarse" channels) $(\mu \mathrm{V})$ & 0 & -511 & -11 & 338 \\
& 120 & -591 & -12 & 447 \\
Differential voltage 2 h drift ("fine" channels) $\left(\mu \mathrm{V}_{\mathrm{p}-\mathrm{p}}\right)$ & 0 and 120 & 77 & 104 & 127 \\
Differential voltage 2 h drift ("coarse" channels) $\left(\mu \mathrm{V}_{\mathrm{p}-\mathrm{p}}\right)$ & 0 and 120 & 327 & 386 & 487 \\
dc CMR (ppm) & 120 & $-22,9$ & $-0,9$ & 20,6 \\
\hline \hline
\end{tabular}

${ }^{\mathrm{a}} V_{\text {out }}$ is the common-mode output voltage at zero differential output voltage $\left(V_{\text {out } A}=V_{\text {out } B}\right)$. 


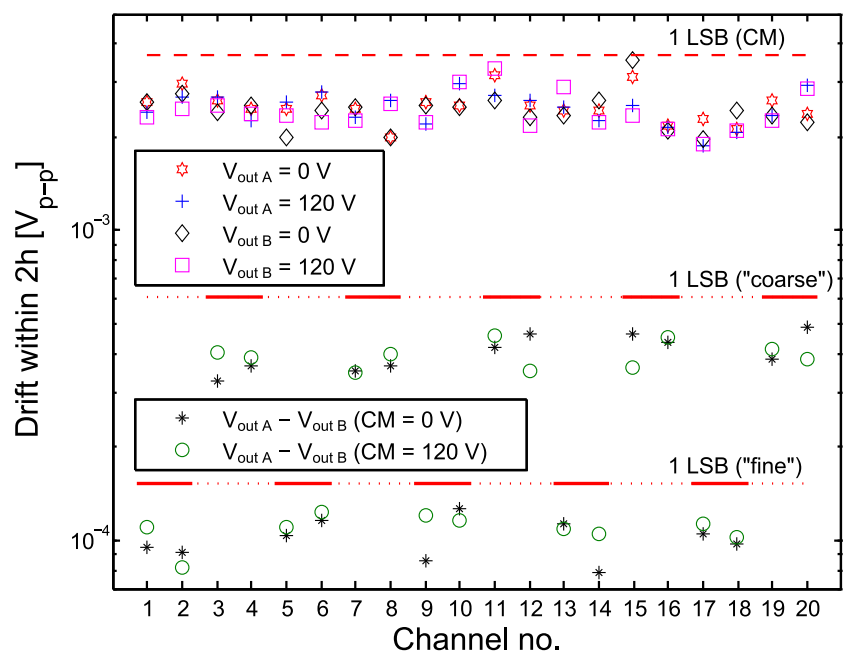

FIG. 6. The drift behavior of ground-referenced and differential voltages measured for $2 \mathrm{~h}$ from all channels.

warm-up time dramatically, without affecting the settled-state drift characteristics. All measurements were taken in a regular room environment without temperature regulation.

Figure 5 shows time plots of the voltages measured quasisimultaneously from a single unit. The recorded data were used to estimate the warm-up drifts, as well as peak-to-peak excursions of settled voltages. Statistical results for all channels are given in Table III. Warm-up values were measured as a difference between averaged measurements in the first $60 \mathrm{~s}$ and measurements taken after $3600 \mathrm{~s}(1 \mathrm{~h})$. Peak-to-peak drift values were measured starting $1 \mathrm{~h}$ after turn-on for a duration of 2 h. Results of settled-state drift measurements are given for all channels (Fig. 6) and for the channels within unit no. 1 (Fig. 7).

Noise was measured using an Agilent 89441A FFT analyzer and a self-made amplifier based on a broadband, lownoise operational amplifier ${ }^{31}$ with a voltage gain $\mathrm{G}=100$ and input-referred white noise level $\mathrm{e}_{\mathrm{n}} \approx 2,5 \mathrm{nV} \mathrm{Hz}^{-1 / 2}$. It was necessary to use it in order to reduce the pickup of EMI, which is

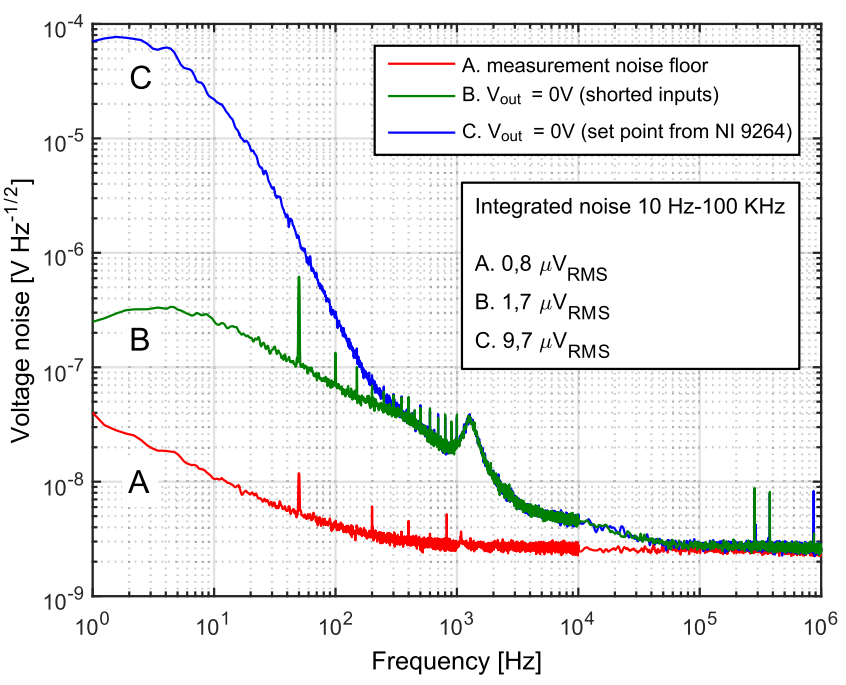

FIG. 8. Noise measured from a single output $\left(V_{\text {out }}=0 \mathrm{~V}\right)$ referenced to ground.

omnipresent in regular building environments. Results of the noise measurements for $V_{\text {out }}=0 \mathrm{~V}$ are shown in Fig. 8. Values for the integrated noise in the $10 \mathrm{~Hz}-100 \mathrm{kHz}$ band were obtained by numeric integration of the spectral density.

\section{DISCUSSION}

We present the design of a scalable, all-linear system capable of producing voltage pairs with independently settable common-mode and differential-mode levels. The system employs feedback loops to stabilize the differential voltages in a ground-referenced circuit topology that allows numerous channels to operate on the same power supply lines. Galvanic ground loops are prevented by having "floating" power supplies. To our knowledge, this is the first publication dealing with a multichannel dc source of moderately high voltages, specifically designed for high-precision experiments in Paul traps.

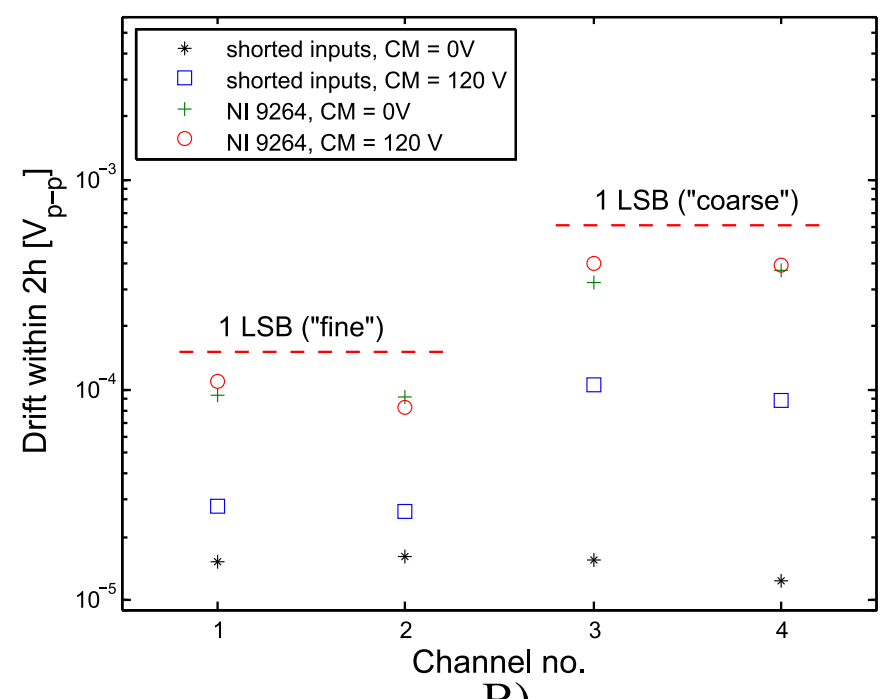

B)

FIG. 7. A comparison of the stability of output voltages measured from unit no. 1 with set point inputs driven by NI 9264 and more stable sources (shorted $V_{D I F F}$ inputs and $V_{C M}=+10 \mathrm{~V}$ from a LT1027-based source). (a) Ground-referenced voltages. (b) Differential voltages. 
Measurement results show excellent stability characteristics. The peak-to-peak excursions of the voltages after the initial warm-up time are all within $1 \mathrm{LSB}$, yielding true dc stability of 15 bits for the common-mode and 16 bits for the differential voltages. Furthermore, the stability of the differentially measured voltages does not depend strongly on the common-mode setting.

It is evident from the measurement data that the performance is limited by the set point voltages generated by the NI 9264 units. Figure 6 and Table III show clearly the consistent difference between the differential "fine" and "coarse" channels due to the 4:1 division of the differential setting voltage $V_{D I F F}$. The same effect is also visible for the output voltages measured against ground. There, the control voltage $V_{C M}$ is amplified by the composite amplifier $(G=12)$, which also leads to amplification of its drift by the same factor. Since the $V_{C M}$ voltages use only the positive half of the $\pm 10 \mathrm{~V}$ range of NI 9264 outputs, the setting resolution is reduced by one bit, but the drift is not scaled, leading to a factor-of-6 higher drift value than in "coarse" differential channels.

The performance of the system could be improved by using DACs with a more stable reference voltage source. In order to estimate the practical limits, we took measurements with shorted inputs and with a quiet, stable $10 \mathrm{~V}$ source (Fig. 7). They reveal that ground-referenced voltages can have much higher stability, perhaps on the order of $1 \mathrm{ppm}$ (20 bits) (Fig. 7(a)). The differential voltages, however, show an increased drift at the highest common-mode setting (Fig. 7(b)), limiting the stability to the 16-bit level. This additional drift is due to Joule heating of the integrated resistors within the difference amplifiers, which becomes significant at high commonmode voltages. It can be seen also in the differential voltage warm-up data summarized in Table III. A possible way of improvement is to use the DFN package variant of LT6375A, which has about 3 times lower junction-to-ambient thermal resistance $R_{\theta J A} .{ }^{30}$ The part was not available to us during the prototyping and layout stage of the project.

As expected, at low frequencies, noise from the NI 9264 unit dominates (Fig. 8). Above a few hundred $\mathrm{Hz}$, due to low-pass filtering, it reaches the intrinsic noise level of the system. Already below $50 \mathrm{kHz}$, the noise spectral density at the filtered outputs drops below $3 \mathrm{nV} \mathrm{Hz}^{-1 / 2}$, which is the measurement noise floor. At the trap electrodes, this noise is further suppressed by $>60 \mathrm{~dB}$ by the last filtering stage (Fig. 2). Thus, the system noise at these frequencies is expected to be lower than the estimated Johnson noise in the bulk resistance of the trap electrodes $\left(e_{n}=40 \mathrm{pV} \mathrm{Hz}^{-1 / 2}\right.$ for $R=0.1 \Omega$ at $\mathrm{T}=300 \mathrm{~K}$ ). We note the illustrative nature of this rough estimate, as a complete model of Johnson noise should include not only the ohmic resistance of the electrodes but also losses in the filter capacitors and the dielectric material of the trap. ${ }^{9}$

If necessary, the ranges of all voltages can be extended. The common-mode voltage limits are set by the supply voltages of the PA340CC (up to $350 \mathrm{~V}$ ) and the commonmode range of LT6375 $( \pm 270 \mathrm{~V})$. The differential voltages can be extended by taking advantage of the flexibility of the LT6375, which can be configured for lower-than-unity gain by rewiring the connections to its built-in resistors. ${ }^{30}$
However, the input resistance of the difference amplifier should be considered, especially in configurations where it is lowered or the common-mode voltage is increased. Under such conditions, Joule heating may limit the achievable operating ranges and the stability of the differential voltages. In such cases, one may consider using difference amplifiers with higher input resistance. ${ }^{32}$

The presented system is designed for Paul traps but could be easily modified and used for other applications. It is particularly suitable in situations where a large number of paired electrodes need stable voltage biasing or where two separate feedback loops implement a "fine-coarse" control scheme. In order to modify our system for dynamic operation, one must tune the compensation of the composite amplifiers and the integrator time-constant within the differential loop.

\section{ACKNOWLEDGMENTS}

We acknowledge support from DFG through Grant Nos. CRC 1128 geo- $Q$, project A03 and CRC 1227 DQ-mat, projects B03 and B05.

${ }^{1}$ D. J. Wineland, C. Monroe, W. M. Itano, D. Leibfried, B. E. King, and D. M. Meekhof, "Experimental issues in coherent quantum-state manipulation of trapped atomic ions," J. Res. Natl. Inst. Stand. Technol. 103(3), 259-328 (1998)

${ }^{2}$ A. Ludlow, M. Boyd, J. Ye, E. Peik, and P. O. Schmidt, "Optical atomic clocks," Rev. Mod. Phys. 87(2), 637-701 (2015).

${ }^{3}$ N. Huntemann, B. Lipphardt, C. Tamm, V. Gerginov, S. Weyers, and E. Peik, "Improved limit on a temporal variation of $\mathrm{mp} / \mathrm{me}$ from comparisons of $\mathrm{Yb}^{+}$and Cs atomic clocks," Phys. Rev. Lett. 113(21), 210802-1-210802-5 (2014).

${ }^{4}$ R. M. Godun, P. B. R. Nisbet-Jones, J. M. Jones, S. A. King, L. A. M. Johnson, H. S. Margolis, K. Szymaniec, S. N. Lea, K. Bongs, and P. Gill, "Frequency ratio of two optical clock transitions in ${ }^{171} \mathrm{Yb}^{+}$and constraints on the time variation of fundamental constants," Phys. Rev. Lett. 113(21), 210801-1-210801-5 (2014).

${ }^{5}$ H. Häffner, C. F. Roos, and R. Blatt, "Quantum computing with trapped ions," Phys. Rep. 469(4), 155-203 (2008).

${ }^{6}$ R. Blatt and C. F. Roos, "Quantum simulations with trapped ions," Nat. Phys. 8(4), 277-284 (2012).

${ }^{7}$ W. Paul, "Electromagnetic traps for charged and neutral particles," Rev. Mod. Phys. 62(3), 531-540 (1990).

${ }^{8}$ P. K. Ghosh, Ion Traps, 1st ed. (Clarendon Press, Oxford, New York, 1996).

${ }^{9}$ M. Brownnutt, M. Kumph, P. Rabl, and R. Blatt, "Ion-trap measurements of electric-field noise near surfaces," Rev. Mod. Phys. 87(4), 1419-1482 (2015).

${ }^{10}$ K. Pyka, N. Herschbach, J. Keller, and T. E. Mehlstäubler, “A high-precision segmented Paul trap with minimized micromotion for an optical multi-ion clock," Appl. Phys. B 114(1), 231-241 (2014).

${ }^{11}$ P. O. Schmidt, T. Rosenband, C. Langer, W. M. Itano, J. C. Bergquist, and D. J. Wineland, "Spectroscopy using quantum logic," Science 309(5735), 749-752 (2005).

${ }^{12}$ Y. Wan, F. Gebert, J. B. Wübbena, N. Scharnhorst, S. Amairi, I. D. Leroux, B. Hemmerling, N. Lörch, K. Hammerer, and P. O. Schmidt, "Precision spectroscopy by photon-recoil signal amplification," Nat. Commun. 5, 3096 (2014).

${ }^{13}$ F. Wolf, Y. Wan, J. C. Heip, F. Gebert, C. Shi, and P. O. Schmidt, "Nondestructive state detection for quantum logic spectroscopy of molecular ions," Nature 530(7591), 457-460 (2016).

${ }^{14}$ D. Kielpinski, C. Monroe, and D. J. Wineland, "Architecture for a large-scale ion-trap quantum computer," Nature 417(6890), 709-711 (2002).

${ }^{15}$ M. T. Baig, M. Johanning, A. Wiese, S. Heidbring, M. Ziolkowski, and C. Wunderlich, "A scalable, fast, and multichannel arbitrary waveform generator," Rev. Sci. Instrum. 84, 124701 (2013).

${ }^{16}$ D. J. Berkeland, J. D. Miller, J. C. Bergquist, W. M. Itano, and D. J. Wineland, "Minimization of ion micromotion in a Paul trap," J. Appl. Phys. 83(10), 5025-5033 (1998). 
${ }^{17}$ J. Keller, H. L. Partner, T. Burgermeister, and T. E. Mehlstäubler, "Precise determination of micromotion for trapped-ion optical clocks," J. Appl. Phys. 118(10) (2015).

${ }^{18}$ C. K. Boggs, A. D. Doak, and F. L. Walls, "Measurement of voltage noise in chemical batteries," in Proceedings of the 1995 IEEE International Frequency Control Symposium, 1995.

${ }^{19}$ D. B. Pinegar, K. Blaum, T. P. Biesiadzinski, S. L. Zafonte, and R. Van Dyck, Jr., "Stable voltage source for Penning trap experiments," Rev. Sci. Instrum. 80(6), 064701 (2009).

${ }^{20} \mathrm{P}$. Horowitz and W. Hill, The Art of Electronics, 3rd ed. (Cambridge University Press, 2015)

${ }^{21}$ A. Wagner, S. Sturm, B. Schabinger, K. Blaum, and W. Quint, “A batterybased, low-noise voltage source," Rev. Sci. Instrum. 81(6) (2010).

${ }^{22}$ J. Williams, "AN118. High voltage, low noise, DC/DC converters," Linear Technol. (2008).

${ }^{23}$ C. Böhm, S. Sturm, A. Rischka, A. Dörr, S. Eliseev, M. Goncharov, M. Höcker, J. Ketter, F. Köhler, D. Marschall, J. Martin, D. Obieglo, J. Repp, C. Roux, R. X. Schüssler, M. Steigleder, S. Streubel, T. Wagner, J. Westermann, V. Wieder, R. Zirpel, J. Melcher, and K. Blaum, “An ultra-stable voltage source for precision Penning-trap experiments," Nucl. Instrum. Methods Phys. Res., Sect. A 828, 125-131 (2016).

${ }^{24}$ M. Lee, "AN82. Understanding and applying voltage references," Linear Technol. (1999).

${ }^{25}$ J. Dostal, Operational Amplifiers, 2nd ed. (Butterworth-Heinemann, Stoneham, MA, 1993).

${ }^{26} \mathrm{NI} 9264 \pm 10 \mathrm{~V}$, Analog Output, $25 \mathrm{kS} / \mathrm{s} / \mathrm{ch}, 16 \mathrm{Ch}$ Module, National Instruments, 2014.

${ }^{27}$ PA340CC - High voltage operational amplifier, APEX Microtechnology, 2013.

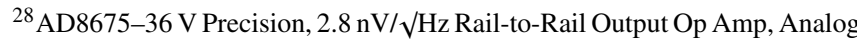
Devices, 2012

${ }^{29}$ F. Seifert, Resistor current noise measurements. LIGO-T0900200-v1, 2009.

${ }^{30}$ LT6375 \pm 270 V Common Mode Voltage Difference Amplifier, Linear Technology, 2016.

${ }^{31}$ OPA820-Unity-Gain Stable, Low-Noise, Voltage-Feedback Operational Amplifier, Texas Instruments, 2008.

${ }^{32}$ AD8479-Very High Common-Mode Voltage Precision Difference Amplifier, Analog Devices, 2016. 